\title{
PERCEPCIÓN DE CUALIDADES RESTAURADORAS DE LOS ESPACIOS ESCOLARES DE BACHILLERATO EN XALAPA, MÉXICO
}

\author{
Aurora de Jesús Mejía-Castillo ${ }^{1}$, Ana Delia López-Suárez ${ }^{1}$, Cesáreo Estrada Rodríguez ${ }^{2}$, Roberto Lagunes-Córdoba ${ }^{{ }^{*}}$ \\ ${ }^{1}$ Instituto de Investigaciones Psicológicas, Universidad Veracruzana. Xalapa, México, \\ ${ }^{2}$ Facultad de Psicología, Universidad Nacional Autónoma de México. México, D.F.
}

Recibido, septiembre $3 / 2015$

Concepto evaluación, mayo 17/2016

Aceptado, mayo 9/2016
Referencia: Mejía-Castillo, A.J., López-Suárez, A. D., Estrada Rodríguez, C. \& Lagunes-Córdoba, R. (2016). Percepción de cualidades restauradoras de los espacios escolares de bachillerato en Xalapa, México. Acta Colombiana de Psicología, 19(2), 199209. DOI: 10.14718/ACP.2016.19.2.9

Resumen

\begin{abstract}
El presente estudio se llevó a cabo con estudiantes de centros educativos de nivel bachillerato en Xalapa, México, y tuvo el objetivo de identificar y comparar las cualidades restauradoras percibidas en sus espacios escolares de acuerdo con la Teoría de la Restauración de la Atención. Se trabajó con una muestra no probabilística de 706 estudiantes de siete bachilleratos. Se recopilaron datos mediante una encuesta sociodemográfica y la Escala del Potencial Restaurador de los Espacios Escolares (EPREE). Los resultados mostraron que las áreas verdes de las escuelas eran los espacios de descanso con mayores cualidades restauradoras, y que los lugares de descanso favoritos poseían más cualidades restauradoras que los no favoritos. Los alumnos varones, los de más edad y aquellos cuya madre (jefe de familia) tenía niveles inferiores de estudios reportaron una percepción más positiva de los espacios escolares. Las escuelas más grandes y con áreas verdes fueron mejor valoradas en las dimensiones de fascinación, posibilidad de estar alejado y extensión. Los resultados confirmaron las propuestas centrales de la teoría de la restauración de la atención en el contexto de los espacios escolares.

Palabras clave: restauración psicológica, escenario educativo, ambiente escolar, estudiantes, psicología ambiental.
\end{abstract}

\section{PERCEIVED RESTORATIVENESS IN SCHOLAR SPACES OF HIGH SCHOOLS IN XALAPA CITY, MÉXICO}

\begin{abstract}
The present study was carried out with high school students from Xalapa, Mexico. Its target was to identify and compare the perceived restorativeness in their school environments according to the attention restoration theory. This work is based on a non-probabilistic sample of 706 students from seven high schools. All data were compiled by a sociodemographic survey and the Scale of the Restorative Potential of School Spaces (EPREE is the Spanish acronym). The results proved that green areas were the rest spaces with major restorative qualities and that the student's favorite rest places possessed more restorative qualities than other places. Male students, older students and those whose mother (head of the family) was less educated reported a more positive perception of the school non classroom spaces. The bigger schools with green areas obtained a higher evaluation for their dimensions of fascination, being away and extent. The results confirmed the main propositions of the attention restoration theory in high school settings.
\end{abstract}

Keywords: psychological restoration, educational settings, school environments, students, environmental psychology.

\footnotetext{
Instituto de Investigaciones Psicológicas. Universidad Veracruzana. Av. Luis Castelazo Ayala S/N. Col. Industrial Ánimas. C.P. 91190. Xalapa, Ver. México.Tel. 00(52)-228-841-8900.rlc.academico@yahoo.com.mx.
} 


\title{
PERCEPÇÃO DE QUALIDADES RESTAURADORAS DOS ESPAÇOS ESCOLARES DE ENSINO MÉDIO NA CIDADE DE XALAPA, MÉXICO
}

\begin{abstract}
Resumo
Este artigo foi realizado com estudantes de centros educativos de ensino médio em Xalapa (México) e teve como objetivo identificar e comparar as qualidades restauradoras percebidas em seus espaços escolares de acordo com a Teoria da Restauração da Atenção. Trabalhou-se com uma amostra não probabilística de 706 estudantes de sete turmas de ensino médio. Coletaramse dados mediante uma enquete sociodemográfica e a Escala do Potencial Restaurador dos Espaços Escolares (EPREE). Os resultados mostraram que as áreas verdes dos centros eram os espaços de descanso com maiores qualidades restauradoras e que os lugares de descanso preferidos pelos estudantes possuíam mais qualidades restauradoras do que os não preferidos. Os estudantes do gênero masculino, os de mais idade e aqueles cuja mãe (cabeça de família) tinha níveis inferiores de escolaridade relataram uma percepção mais positiva dos espaços escolares. Os maiores centros e com áreas verdes tiveram melhores avaliações nas dimensões de fascinação, possibilidade de estar isolado e extensão. Os resultados confirmaram as propostas centrais da Teoria da Restauração da Atenção no contexto dos espaços escolares.

Palavras-chave: restauração psicológica, cenário educativo, ambiente escolar, estudantes, psicologia ambiental.
\end{abstract}

\section{INTRODUCCIÓN}

El mundo es una fuente constante de estímulos para el ser humano. La sobrecarga de información del ambiente demanda a las personas una atención directa, es decir, llevar a cabo un esfuerzo mental prolongado para realizar las actividades cotidianas requeridas, evadiendo distractores que pueden interferir en sus tareas (Kaplan y Kaplan, 2009). Al cabo del tiempo, este proceso ocasiona fatiga mental, que repercute en las habilidades de los sujetos para desenvolverse a niveles cognitivo y afectivo, de manera óptima.

Las personas requieren espacios o escenarios que les permitan restaurar sus capacidades psicológicas para continuar con sus labores eficazmente. De esta manera, se entiende como ambiente restaurador aquel escenario que favorece la recuperación psicológica de los individuos (Kaplan y Talbot, 1983). Por su parte, la restauración psicológica consiste en la complejidad de procesos que permiten al organismo recuperar las capacidades y los recursos funcionales que se han visto disminuidos por las demandas del ambiente (Hartig y Staats, 2003).

La Teoría de la Restauración de la Atención (TRA) describe algunas cualidades que permiten a las personas restaurarse psicológicamente, después de enfrentarse a situaciones que generan fatiga mental. Desde esta perspectiva, la percepción de cualidades restauradoras (traducido del inglés restorativeness) o del potencial restaurador del ambiente, involucra cuatro dimensiones fundamentales (Kaplan y Kaplan, 1989):

a) Fascinación: conjunto de características percibidas que atraen la atención de la persona. Se trata de una atención involuntaria, es decir, que no exige un sobreesfuerzo mental. b) Estar alejado: conjunto de características percibidas que le permiten al individuo distanciarse, física o psicológicamente, de aquello que le exige atención dirigida.

c) Compatibilidad: percepción de que el ambiente coincide con los propósitos de quien lo experimenta.

d) Extensión: cualidad del ambiente que invita a explorar más allá de lo que se percibe de manera inmediata (Scopelliti y Giulliani, 2004). Dentro de esta dimensión se encuentra la coherencia o capacidad de percibir claridad y orden en ese espacio (Kaplan, 1995). Aunque Kaplan considera a la coherencia como un elemento de la extensión, Martínez-Soto y Montero (2010) encontraron que forma una dimensión adicional, separada del alcance (característica de la extensión), en contextos mexicanos.

En los estudios sustentados en la TRA se ha comprobado que en los ambientes naturales donde existe la presencia de vegetación y agua se perciben mayores cualidades restauradoras (Hernández e Hidalgo, 2005; Kaplan y Kaplan, 1989). Sin embargo, la experiencia restauradora no sucede solo en la presencia de ambientes naturales (Hartig, Mang y Evans, 1991), y no todos los ambientes de este tipo contribuyen a la restauración, principalmente cuando existen otras variables que influyen en los sujetos que experimentan el escenario, como la percepción de peligro o la falta de agrado (Van den Berg, Hartig y Staats, 2007; Van den Berg y Heijne, 2005).

Otro hallazgo encontrado reiteradamente es que los lugares preferidos presentan mayores niveles de percepción de cualidades restauradoras (Korpela, Kytta y Hartig, 2002; Martínez-Soto y Montero, 2010; Wilkie y Stavridou, 2013). A su vez, los efectos de la restauración en las personas se pueden observar en cambios favorables en el estado de ánimo, disminución de la activación fisiológica y el mejo- 
ramiento en el desempeño de tareas que requieren atención directa (Hartig y Staats, 2003).

En las escuelas, los alumnos se enfrentan a diversas fuentes de estrés ambiental (ruido, tamaño de la escuela, disposición del mobiliario, diseño, entre otros), que impactan negativamente el bienestar de los estudiantes (Mejía-Castillo, 2010) y coadyuvan a la disminución de su rendimiento académico. Además, las propias instalaciones tienen características físicas que pueden favorecer la recuperación psicológica de los alumnos. Algunos estudios reportan que incorporar elementos restauradores en las escuelas (principalmente plantas o árboles) se relaciona con mayores sentimientos de comodidad y amabilidad, menores niveles de ausentismo y sanciones por mala conducta (Han, 2009); mejor rendimiento y eficiencia terminal de los estudiantes (Matsuoka, 2010); mayor proambientalidad (Collado y Corraliza, 2013), así como mayor bienestar fisiológico y psicológico de los estudiantes (Kels, Evans y Röderer, 2013).

A pesar de la trascendencia de estos estudios en el ámbito mundial, en México no se ha encontrado información sobre las cualidades restauradoras de los escenarios escolares. Los estudios hasta el momento se han centrado en algunos de los factores del ambiente, como el ruido en aulas de nivel primaria (Estrada, 2007; Estrada y Méndez, 2010) o la inadecuada disposición del mobiliario en preescolar (Urbina, 1981) y sus consecuencias negativas, tanto cognitivas como conductuales.

Por otra parte, los estudios fuera de México que se basan en las dimensiones de la TRA se centran principalmente en las cualidades físicas del ambiente, dejando de lado las características del contexto social que podrían estar vinculadas al proceso de restauración. La mayoría de los estudios utiliza adaptaciones de la Escala de cualidades restauradoras percibidas (Perceived Restorativeness Scale) de Hartig, Korpela, Evans y Garling (1997), la cual es una escala adecuadamente confiable y válida de las dimensiones de la TRA, pero está pensada para evaluar diversos ambientes naturales y construidos, por lo que no incorpora algunos elementos particulares de la realidad del contexto escolar.

En un estudio cualitativo con estudiantes de bachillerato, Mejía-Castillo y Lagunes-Córdoba (2015) encontraron que pese a la falta de vegetación, áreas verdes y deterioro de algunas escuelas, los estudiantes identifican ciertas cualidades restauradoras (relacionadas con las dimensiones de la TRA) que incorporan las posibilidades de interacción y contacto social.

La revisión teórica y los escasos avances en la investigación de cualidades restauradoras de los escenarios educativos mexicanos, ponen en evidencia la necesidad de conocer mejor el contexto.

El presente trabajo se realizó en centros educativos de nivel bachillerato, en la ciudad de Xalapa, Veracruz, México, y tuvo como objetivos:

1. Identificar los espacios de descanso que utilizaban los estudiantes de bachillerato en sus centros escolares.

2. Determinar las diferencias en la percepción de cualidades restauradoras en estos espacios, y establecer comparaciones y asociaciones en la percepción de estas cualidades con respecto a variables sociodemográficas de los alumnos, preferencia por el lugar, y características físicas de las escuelas (presencia de áreas verdes y tamaño de las escuelas).

\section{MÉTODO}

\section{Participantes}

Se integró una muestra no probabilística por conveniencia de 706 estudiantes ( $49 \%$ hombres y $51 \%$ mujeres), de primer y segundo año escolar, de siete escuelas de nivel bachillerato general de Xalapa, Veracruz, México. El rango de edad de los participantes fue de 15 a 19 años, con una media de 16 años. Los estudiantes participantes fueron seleccionados por las propias autoridades escolares en función de sus horarios y disponibilidad de espacios.

Los criterios de inclusión consistieron en que los alumnos se encontraran inscritos en la escuela en el momento del estudio, y que contestaran los cuestionarios de manera voluntaria y completa. Se excluyeron los estudiantes que estaban en los últimos tres semestres, debido a que estos cursan opciones tecnológicas que podrían hacerlos tener acceso a espacios restauradores diferentes que no estuvieran al alcance de los demás estudiantes.

\section{Escenarios}

El estudio se llevó a cabo en la ciudad de Xalapa, capital del estado de Veracruz, que se encuentra al este de la República Mexicana. De acuerdo con el Censo de Población y Vivienda (Instituto Nacional de Estadística y Geografía, 2010), cuenta con 457.614 habitantes. Dentro de este contexto, la educación de nivel medio superior (bachillerato) se imparte por escuelas públicas y privadas que se organizan en distintas modalidades (bachillerato general, bachillerato tecnológico y educación profesional técnica).

Las siete escuelas ubicadas en la ciudad de Xalapa conformaron los escenarios de estudio, y presentaron características muy diversas, las cuales se presentan en la Tabla 1. 
Tabla 1.

Características generales de las escuelas estudiadas

\begin{tabular}{|c|c|c|c|}
\hline Escuela & Características generales & $\begin{array}{l}\text { Extensión total } \\
\text { aproximada* }\end{array}$ & $\begin{array}{l}\text { Extensión de área verde } \\
\text { aproximada }^{*}\end{array}$ \\
\hline Escuela A & $\begin{array}{l}\text { Se ubica en el centro de la ciudad. Posee un edificio de dos } \\
\text { pisos y un patio central. }\end{array}$ & 500 metros cuadrados & $\begin{array}{l}\text { No hay áreas verdes, solo } \\
\text { macetas. }\end{array}$ \\
\hline Escuela B & $\begin{array}{l}\text { Se ubica en el centro de la ciudad. Posee tres edificios, un patio } \\
\text { central y una cancha. }\end{array}$ & 2.226 metros cuadrados & $\begin{array}{l}\text { No hay áreas verdes, solo } \\
\text { macetas. }\end{array}$ \\
\hline Escuela C & $\begin{array}{l}\text { Se ubica en el centro de la ciudad. Posee un edificio de tres } \\
\text { niveles y al centro un patio cuadrangular que se divide en dos } \\
\text { canchas. }\end{array}$ & 2.475 metros cuadrados & $\begin{array}{l}\text { No hay áreas verdes, solo } \\
\text { macetas. }\end{array}$ \\
\hline Escuela D & $\begin{array}{l}\text { Se ubica en la periferia de la ciudad. El terreno es compartido } \\
\text { por una secundaria y una licenciatura. El área cuenta con cinco } \\
\text { edificios, de los cuales dos son de uso del bachillerato. Cuenta } \\
\text { con una cancha y estacionamientos. }\end{array}$ & $\begin{array}{l}12.800 \text { metros } \\
\text { cuadrados }\end{array}$ & $\begin{array}{l}4.715 \text { metros cuadrados } \\
\text { ( } 36.84 \% \text { de la extensión total) }\end{array}$ \\
\hline Escuela E & $\begin{array}{l}\text { Se encuentra en los límites del centro de la ciudad. Posee dos } \\
\text { edificios, uno de un nivel y otro de tres niveles. Cuenta cancha } \\
\text { y estacionamientos. }\end{array}$ & $\begin{array}{l}11.658 \text { metros } \\
\text { cuadrados }\end{array}$ & $\begin{array}{l}5.397 \text { metros cuadrados } \\
(46.3 \% \text { de la extensión total })\end{array}$ \\
\hline Escuela F & $\begin{array}{l}\text { Ubicada en la periferia de la ciudad. Posee cuatro edificios de } \\
\text { un solo nivel. Tiene un patio y una cancha. }\end{array}$ & $\begin{array}{l}19.700 \text { metros } \\
\text { cuadrados }\end{array}$ & $\begin{array}{l}11.242 \text { metros cuadrados } \\
(57.07 \% \text { de la extensión total })\end{array}$ \\
\hline Escuela G & $\begin{array}{l}\text { Ubicada en la periferia de la ciudad. Posee seis edificios, de uno } \\
\text { y dos niveles. Tiene un patio central, una cancha y un campo } \\
\text { de futbol. }\end{array}$ & $\begin{array}{l}20.000 \text { mil metros } \\
\text { cuadrados }\end{array}$ & $\begin{array}{l}18.196 \text { metros cuadrados } \\
(90.98 \% \text { de la extensión total })\end{array}$ \\
\hline
\end{tabular}

Nota: ${ }^{M}$ Medida obtenida a través de Google Earth Pro (2015).

\section{Instrumentos}

Cédula de datos sociodemográficos y generales. Formato de datos generales que integra: escuela de procedencia, edad, sexo, promedio, lugar de descanso, lugar favorito de los estudiantes en la escuela y nivel de escolaridad de los padres o tutores de los cuales dependieran económicamente. Además, se reservaron unas líneas para que los estudiantes describieran las características de su espacio de descanso, y las actividades que podían realizar en ellos.

Escala del Potencial Restaurador de los Espacios Escolares (EPREE). Escala psicométrica construida y validada para los fines de este estudio y las características propias de los espacios escolares (no publicada). La escala EPREE presenta reactivos construidos específicamente para la evaluación de las dimensiones del potencial restaurador de espacios escolares. Consiste en un formato tipo Likert pictórico (Reyes-Lagunes, 1993) con cinco opciones de respuesta, donde cada reactivo presenta al costado cinco cuadros (del más grande al más pequeño), cuyas opciones equivalen a la escala Likert tradicional (totalmente de acuerdo a totalmente en desacuerdo). La escala consta de 20 reactivos, divididos en cinco dimensiones: fascinación $(\alpha=0.90)$, compatibilidad $(\alpha=0.78)$, alejamiento $(\alpha=0.79)$, coherencia $(\alpha=0,71)$ y extensión $(\alpha=0.66)$. La estructura factorial obtuvo índices adecuados de ajuste en el análisis factorial confirmatorio $(\mathrm{CFI}=0.95, \mathrm{GFI}=0.945$, $\mathrm{TLI}=0.94$, RMSEA $=0.05$ ), con una confiabilidad total de 0.86 y una varianza común explicada de 54,6\%.

Mapas de escuelas. Se utilizaron mapas obtenidos a través del programa Google Earth Pro 2015 para calcular la extensión total y el porcentaje visualizado de áreas verdes en cada escuela.

\section{Procedimiento}

Se realizó una solicitud formal a la Dirección General de Educación Media Superior del Estado de Veracruz, México. Posterior a ello, se tuvieron entrevistas con las autoridades de cada plantel para dar a conocer las situaciones del estudio. Las aplicaciones se realizaron en junio de 2015, con el apoyo de un total de seis encuestadores, quienes fueron previamente capacitados.

Los aplicadores explicaron al inicio las instrucciones al grupo. La aplicación tuvo una duración aproximada de 20 minutos, tiempo durante el cual los encuestadores estuvieron pendientes de aclarar dudas y términos complicados para los estudiantes. El análisis de datos se llevó a cabo a través del programa SPSS versión 20.0. 


\section{Análisis estadístico}

Se utilizaron frecuencias, porcentajes y estadística descriptiva para analizar las características generales de la población. Las diferencias entre grupos para las variables categóricas se determinaron mediante la prueba de $X^{2}$, y por la falta de normalidad se utilizaron las pruebas de KruskallWallis y U de Mann-Whitney en las variables continuas.

Como medidas de asociación se utilizaron los coeficientes de contingencia para variables categóricas, y se usó correlación de Spearman para variables continuas.

\section{RESULTADOS}

A continuación se presentan las características generales de la muestra obtenida, el reporte de los espacios de descanso más utilizados por escuela, junto con las cualidades restauradoras percibidas en ellos y las diferencias de percepción de cualidades restauradoras de acuerdo con las características sociodemográficas de los alumnos. Posteriormente, se comparan las cualidades restauradoras percibidas cuando se trataba del lugar favorito de los alumnos y cuando no lo era, y se finaliza comparando las cualidades restauradoras percibidas para escuelas de diferentes tamaños y con diferente proporción de áreas verdes con respecto a su extensión total.

\section{Características generales de la población}

La tabla 2 presenta los datos generales de la población encuestada, que incluyen para cada una de las escuelas: edad de los participantes, porcentaje por sexo, grado de estudios del jefe de familia (padre, madre u otro) y promedio de calificaciones de los estudiantes. En cuanto a la distribución de sexo, en algunas escuelas la muestra de hombres fue superior a la de mujeres, y en otras, el caso fue a la inversa.

La media de edades presentó diferencias significativas. La escuela $\mathrm{F}$ tuvo la media más alta de todas. Los promedios de calificación también presentaron diferencias significativas, con las escuelas $\mathrm{C}$ y D en los promedios más altos. Es destacable el hecho de que los estudiantes provenientes de las escuelas céntricas de la ciudad (principalmente escuela B y C), dependían de padres con niveles educativos superiores.

Tabla 2.

Características sociodemográficas generales

\begin{tabular}{lccccccccc}
\hline \multicolumn{1}{c}{ Variable } & Población total & Escuela A & Escuela B & Escuela C & Escuela D & Escuela E & Escuela F & Escuela G & p \\
\hline Edad \pm SD & $16 \pm 0.75$ & $15.6 \pm 0.53$ & $15.6 \pm 0.49$ & $16 \pm 0.67$ & $15.7 \pm 0.54$ & $16.3 \pm 0.74$ & $16.9 \pm 0.66$ & $16 \pm 0.77$ & $<0.001 \alpha$ \\
Sexo & & & & & & & & & $0.45^{* *}$ \\
Hombre & 341 & 62 & 49 & 51 & 42 & 55 & 37 & 45 & \\
& $(48.5 \%)$ & $(55.9 \%)$ & $(50.5 \%)$ & $(45.5 \%)$ & $(40 \%)$ & $(49.5 \%)$ & $(46.8 \%)$ & $(51.1 \%)$ & \\
Mujer & 361 & 49 & 48 & 61 & 63 & 55 & 42 & 43 & \\
& $(51.4 \%)$ & $(44.1 \%)$ & $(49.5 \%)$ & $(54.5 \%)$ & $(60 \%)$ & $(49.5 \%)$ & $(53.2 \%)$ & $(48.9 \%)$ & \\
Estudios & & & & & & & & & $<0.001^{* *}$ \\
del padre & & & & & & & & & \\
Primaria & $6.8 \%$ & $7.2 \%$ & $1 \%$ & $2.7 \%$ & $7.6 \%$ & $8.1 \%$ & $12.7 \%$ & $10.2 \%$ & \\
Secundaria & $15.8 \%$ & $6.3 \%$ & $9.3 \%$ & $5.4 \%$ & $28.6 \%$ & $17.1 \%$ & $22.8 \%$ & $25 \%$ & \\
Bachillerato & $20.8 \%$ & $21.6 \%$ & $10.3 \%$ & $10.7 \%$ & $26.7 \%$ & $24.3 \%$ & $22.8 \%$ & $30.7 \%$ & \\
Licenciatura & $24.6 \%$ & $27.9 \%$ & $43.3 \%$ & $41.1 \%$ & $12.4 \%$ & $20.7 \%$ & $11.4 \%$ & $10.2 \%$ & \\
Posgrado & $11.7 \%$ & $13.5 \%$ & $20.6 \%$ & $24.1 \%$ & $2.9 \%$ & $8.1 \%$ & $1.3 \%$ & $8 \%$ & \\
Total & $79.7 \%$ & $76.6 \%$ & $84.5 \%$ & $70.9 \%$ & $78.1 \%$ & $78.4 \%$ & $70.9 \%$ & $84.1 \%$ & \\
Estudios & & & & & & & & & $0.001^{* *}$ \\
de la madre & & & & & & & & & \\
Primaria & $7.3 \%$ & $1.8 \%$ & $3.1 \%$ & $2.7 \%$ & $8.6 \%$ & $8.1 \%$ & $20.3 \%$ & $14.1 \%$ & \\
Secundaria & $16.8 \%$ & $15.3 \%$ & $5.2 \%$ & $6.3 \%$ & $25.7 \%$ & $24.3 \%$ & $22.8 \%$ & $26.6 \%$ & \\
Bachillerato & $20.9 \%$ & $23.4 \%$ & $10.3 \%$ & $11.6 \%$ & $24.8 \%$ & $27.9 \%$ & $20.3 \%$ & $39.1 \%$ & \\
Licenciatura & $24.2 \%$ & $29.7 \%$ & $35.1 \%$ & $42 \%$ & $18.1 \%$ & $17.1 \%$ & $12.7 \%$ & $12.5 \%$ & \\
Posgrado & $12.4 \%$ & $18.9 \%$ & $25.8 \%$ & $17.9 \%$ & $5.7 \%$ & $6.3 \%$ & & & \\
Total & $81.5 \%$ & $89.2 \%$ & $79.4 \%$ & $79.7 \%$ & $82.9 \%$ & $83.8 \%$ & $79.7 \%$ & $100 \%$ & \\
Promedio \pm SD & $7.6 \pm 0.91$ & $7.6 \pm 1$ & $7.6 \pm 0.75$ & $7.8 \pm 0.7$ & $7.8 \pm 1.04$ & $7.7 \pm 0.78$ & $7.3 \pm 1.2$ & $7.5 \pm 0.8$ & $0.003^{*}$ \\
\hline
\end{tabular}

Notas: * Prueba de Kruskal-Wallis.

${ }^{* *}$ Prueba de Chi-cuadrado. 
Espacios de descanso más utilizados en las escuelas

Las respuestas dadas por los participantes con respecto a los lugares de descanso que empleaban pudieron agruparse en seis grandes categorías: 1. Salones de clases, 2. Pasillos o balcones afuera del salón, 3. Explanadas, patios o canchas, 4. Áreas verdes, 5. Áreas de comida o cafetería. 6. Otros espacios (bibliotecas, escaleras, entrada de la escuela). Como se observa en la tabla 3 , los espacios de descanso de mayor uso fueron los salones de clase ( $\mathrm{N}=289)$, y los de menor uso fueron las áreas de comida/cafeterías $(\mathrm{N}=26)$.

Lugares de descanso en las escuelas y cualidades restauradoras percibidas

Para el análisis comparativo de las cualidades restauradoras de las categorías de espacios de descanso se utilizó la prueba de Kruskal-Wallis. Fue necesario excluir la categoría 6, debido a la variedad de respuestas que se agruparon dentro de esta, y que reflejaban características muy específicas de las escuelas. Se encontraron diferencias estadísticamente significativas entre lugares de descanso para la percepción de fascinación, compatibilidad, estar alejado, extensión y potencial restaurador total $(p<0.001$ en todos los casos mencionados). La dimensión de coherencia no presentó diferencias significativas $(p=0.244)$.

Para determinar cuáles lugares de descanso presentaban mayor puntuación en cada característica restauradora, se hicieron comparaciones entre los rangos medios de cada una de las categorías de lugares de descanso (agrupando los datos de las siete escuelas), para cada factor de la escala y la escala total, por medio de la prueba U de Mann-Whitney. La Tabla 3 muestra las diferencias estadísticamente significativas encontradas entre los lugares de descanso próximos, según el orden de su rango promedio.

Tabla 3.

Percepción de cualidades restauradoras por lugar de descanso en el conjunto de las siete escuelas (ordenadas con base en el rango promedio)

\begin{tabular}{|c|c|c|c|}
\hline \multirow[t]{3}{*}{ Dimensiones } & Lugares de descanso & $N$ & Rango promedio \\
\hline & Áreas verdes & 83 & 434.57 \\
\hline & Áreas de comida/cafetería & 26 & 398.58 \\
\hline \multirow{6}{*}{ Fascinación } & Explanadas, patios o canchas & 74 & 365.24 \\
\hline & Pasillos/balcones afuera del salón & 150 & 351.95 \\
\hline & Salón & 289 & $281.27^{* *}$ \\
\hline & Total & 670 & \\
\hline & Áreas de comida/cafetería & 26 & 474.98 \\
\hline & Áreas verdes & 83 & 423.16 \\
\hline \multirow{7}{*}{ Estar alejado } & Explanadas, patios o canchas & 74 & 415.94 \\
\hline & Pasillos/balcones afuera del salón & 151 & $359.26^{* *}$ \\
\hline & Salón & 288 & $246.16^{* *}$ \\
\hline & Total & 670 & \\
\hline & Áreas verdes & 83 & 413.31 \\
\hline & Explanadas, patios o canchas & 74 & 412.28 \\
\hline & Pasillos/balcones afuera del salón & 151 & $351.94^{* *}$ \\
\hline \multirow{6}{*}{ Compatibilidad } & Áreas de comida/cafetería & 26 & 334.62 \\
\hline & Salón & 289 & 294.65 \\
\hline & Total & 671 & \\
\hline & Áreas verdes & 83 & 375.25 \\
\hline & Áreas de comida/cafetería & 26 & 355.23 \\
\hline & Explanadas, patios o canchas & 74 & 337.60 \\
\hline \multirow{5}{*}{ Coherencia } & Pasillos/balcones afuera del salón & 151 & 333.66 \\
\hline & Salón & 289 & 331.57 \\
\hline & Total & 671 & \\
\hline & Áreas verdes & 83 & 483.40 \\
\hline & Explanadas, patios o canchas & 74 & 439.59 \\
\hline \multirow{6}{*}{ Extensión } & Pasillos/balcones afuera del salón & 151 & 364.78 \\
\hline & Áreas de comida/cafetería & 26 & $313.56^{* *}$ \\
\hline & Salón & 289 & 258.69 \\
\hline & Total & 671 & \\
\hline & Áreas verdes & 83 & 472.34 \\
\hline & Áreas de comida/cafetería & 26 & 434.67 \\
\hline \multirow{4}{*}{ Potencial restaurador total (escala total) } & Explanadas, patios o canchas & 74 & 406.50 \\
\hline & Pasillos/balcones afuera del salón & 150 & 355.97 \\
\hline & Salón & 288 & $253.80^{* *}$ \\
\hline & Total & 669 & \\
\hline
\end{tabular}

Nota: ${ }^{* *}$ Se reportan únicamente las diferencias significativas $(\mathrm{p}<0,001)$ con respecto a las categorías próximas superiores, según el rango promedio. 
Los resultados también mostraron que las áreas verdes fueron los espacios que presentaron mayor percepción de fascinación, compatibilidad, extensión y potencial restaurador total, mientras que las áreas de comida/cafeterías ocuparon el primer lugar en la dimensión de estar alejado. En cuanto a las dimensiones de compatibilidad y extensión, las explanadas ocuparon el segundo lugar, y en fascinación y potencial restaurador total, lo hicieron las áreas de comida/cafeterías.

Por otra parte, los lugares con menores cualidades restauradoras percibidas fueron los salones de clase. Estos presentaron los puntajes más bajos para todas las dimensiones de la escala y para el potencial restaurador total.

Percepción de cualidades restauradoras con respecto de las características sociodemográficas de los estudiantes

Para conocer si existían diferencias por sexo en la percepción de cualidades restauradoras de los espacios escolares, se realizó un análisis mediante la prueba de U de Mann-Whitney. Se encontró que los hombres perciben mayor fascinación (rango promedio: 374.39 vs. 328.97, $p=0.003$ ), posibilidad de estar alejado (rango promedio: $370.99, v s .332 .07, p=0.011$ ) y potencial restaurador total (368.69 vs. 333.33, $p=0.021$ ). Para las demás dimensiones no se encontraron diferencias estadísticamente significativas.

Con respecto de la edad, el análisis con la prueba de Kruskal-Wallis determinó que existían diferencias significativas en las dimensiones de fascinación $(p=0.005)$, estar alejado ( $p<0.001)$, extensión $(p<0.001)$ y potencial restaurador total. En todos los casos, la media de rangos fue superior para los alumnos de mayor edad. Esto se vio confirmado por el análisis de correlación (cociente de correlación de Spearman), el cual confirmó que existía una correlación baja, pero significativa en la edad de los estudiantes y las puntuaciones en cada una de las dimensiones señaladas (fascinación, $r=0.126$; estar alejado, $r=0.160$; extensión, $r=0.225$; potencial restaurador total, $r=0.163$, con $p<0.001$ en todos los casos).

También se encontraron diferencias significativas con respecto del grado de estudios de la madre como jefa del hogar. Los rangos promedio de puntuación son significativamente menores (prueba de Kruskal-Wallis) para los alumnos cuya madre tenía estudios de nivel primaria o secundaria en las dimensiones de fascinación $(p<0.001)$, coherencia $(p=0.028)$, extensión $(p<0.001)$ y potencial restaurador total $(p<0.001)$. Esto se confirmó por medio del análisis de correlación (coeficiente de Spearman), que encuentra una correlación negativa baja, pero significativa $(p<0.001)$, entre el nivel de estudios de la madre y los puntajes de fascinación $(r=-0.164)$, coherencia $(r=-0.10)$, extensión $(r=-0.136)$ y potencial restaurador total $(r=-0.171)$.

Cuando se analizó el potencial restaurador total utilizando el resto de las variables sociodemográficas estudiadas, no se encontraron diferencias estadísticamente significativas.

Lugares de descanso favoritos vs. no favoritos y cualidades restauradoras asociadas a los mismos

Los lugares de descanso favoritos que se ubicaron dentro de las cinco categorías analizadas en el apartado anterior fueron las explanadas, canchas o patios $(23 \%$ de las respuestas), seguidas por las áreas verdes (14.4\%), los pasillos o balcones afuera del salón (10.5\%) y las áreas de comida o cafetería (9.2\%). Los espacios menos preferidos fueron los salones de clase (9.2\%). Adicionalmente, el $24.6 \%$ de los lugares favoritos se refirieron a espacios diversos, que entraron en la categoría de "otros", tratándose de espacios muy específicos de cada escuela y que fueron poco mencionados como lugares de descanso. Finalmente, el $7.3 \%$ de los participantes que respondieron a esta pregunta, afirmaron no tener un lugar favorito en sus escuelas.

Para conocer si los espacios de descanso coincidían con los lugares favoritos dentro de las escuelas, se realizaron análisis de correlación por categorías. Se obtuvo un coeficiente de contingencia significativo $0.476(p<0.001)$, lo cual permitió concluir que existía una asociación moderada entre los lugares favoritos de los estudiantes y los lugares donde pasaban su tiempo de descanso.

Para determinar si existían diferencias en la percepción de cualidades restauradoras entre los estudiantes que descansaban en su lugar favorito, frente a los que descansaban en un espacio distinto, se realizó el análisis mediante la prueba U de Mann-Whitney. Se encontró que cuando los lugares de descanso utilizados coincidían con los favoritos, tenían mayores niveles de percepción de fascinación $(p<0.001)$, compatibilidad $(p=0.013)$, posibilidad de estar alejado $(p<0.001)$, extensión $(p=0.001)$ y potencial restaurador total $(p<0.001)$. La única dimensión que no presentó diferencias significativas entre ambos grupos fue la de coherencia $(p=n s)$.

Tamaño de la escuela y percepción de cualidades restauradoras

Con el objetivo de conocer si existían diferencias entre escuelas pequeñas y grandes respecto al nivel de percepción del potencial restaurador, éstas se agruparon de acuerdo con su extensión en dos categorías: escuelas pequeñas (menores a $10.000 \mathrm{~m}^{2}$ ) y escuelas grandes (mayores a $10.000 \mathrm{~m}^{2}$ ).

Las escuelas grandes presentaron mayor nivel de fascinación, posibilidad de estar alejado y extensión, en 
comparación de las escuelas pequeñas ( $\mathrm{p}<0,001$ para todos los casos). El potencial restaurador total también fue significativamente mayor en las escuelas grandes $(\mathrm{p}<0.001)$. No se observaron diferencias estadísticamente significativas en las dimensiones de compatibilidad y coherencia ( $\mathrm{p}=\mathrm{ns}$ para cada caso).

Extensión de área verde y percepción de cualidades restauradoras

Como una parte importante de las consideraciones teóricas en las que se basa el presente trabajo, se analizaron las cualidades restauradoras de las escuelas con base en su porcentaje de extensión total de área verde. Para ello, se determinaron cuatro grupos: 1 . Escuelas sin áreas verdes (solo macetas), 2. Escuelas con porcentajes de área verde entre $25 \%$ y $50 \%$ sobre el total de la extensión, 3 . Escuelas con porcentaje de áreas verdes entre el 50\% y 75\%, y 4. Escuelas entre $75 \%$ y $100 \%$ de área verde. De manera global (prueba de Kruskal-Wallis), se encontraron diferencias estadísticamente significativas para las dimensiones de fascinación, estar alejado, extensión y para el potencial restaurador total ( $p<0.001$ para todos los casos).

Las diferencias específicas entre cada grupo se analizaron por medio de la prueba $\mathrm{U}$ de Mann Whitney. Las medias de rangos mostraron que los valores del grupo 1 fueron significativamente menores que las del grupo 2 en las cuatro dimensiones ( $p=0.001$ en todos los casos). Además, en la dimensión de fascinación, se encontró que los puntajes del grupo 3 fueron significativamente mayores a los del grupo 4 ( $p=0.001$ ). Finalmente, el grupo 3 fue significativamente superior al grupo 2 en extensión percibida $(p=0.006)$.

\section{DISCUSIÓN}

Pese a que las escuelas estudiadas poseen características diversas en sus espacios físicos, se pudieron identificar cinco grupos de lugares que los estudiantes mencionan constantemente como espacios donde pasan su tiempo de descanso: áreas verdes, explanadas o canchas, salones, corredores afuera del salón y áreas de comida o cafeterías. Este fue el panorama general, y únicamente tres escuelas (A, B y C) no poseían espacios en la categoría de áreas verdes.

El estudio demuestra que las áreas verdes son los espacios de la escuela donde existe mayor percepción de fascinación, compatibilidad, extensión y potencial restaurador total. Los resultados coinciden con las investigaciones que encuentran que los escenarios naturales poseen mayores cualidades restauradoras (Kaplan y Kaplan, 1989; Hernández e Hidalgo, 2005).
Dos espacios que también presentaron altos niveles de percepción de cualidades restauradoras fueron las áreas de comida y las explanadas/patios o canchas. Para interpretar esto, es importante considerar que las actividades que se realizan en los espacios son importantes para la percepción de estas cualidades. Algunos comentarios recolectados sobre estos espacios lo manifiestan con claridad: "[la explanada es] un lugar abierto con mucha luz y dónde convivir", "[el espacio de la cafetería es] bonito, amplio, tiene mesas y sillas, huele delicioso ahí adentro, un lugar para poder estar con las amigas, comiendo, jugando o durmiendo". Por ello, es preciso que los estudios sobre cualidades restauradoras tomen en cuenta las actividades que las personas pueden realizar, así como los factores físicos y sociales implicados (Kort, Gal y Staats, 2004).

Por otra parte, se encontró que los salones de clase son los espacios escolares que reciben menores puntuaciones en cualidades restauradoras, y ocupan el último lugar entre los espacios preferidos, pero son los lugares donde la mayoría de los alumnos pasa su tiempo libre. Algunos comentarios respecto al salón son los siguientes: "es grande, blanco, sucio, rayado de las paredes"; "es un cubo sin ventanas, pequeño. Predomina el blanco y siempre se siente demasiada gente dentro de él".

De las cinco grandes categorías consideradas para los lugares de descanso, los espacios preferidos por los estudiantes son las explanadas, patios o canchas $(23.9 \%$ de las respuestas), seguidas de las áreas verdes. Estos datos permiten observar que la relación entre el uso de espacios de descanso y su preferencia llega a invertirse en algunos casos, donde el espacio de descanso de mayor uso (salón), también es el menos veces señalado como favorito, y el espacio preferido (explanadas, patios o canchas) ocupa el cuarto lugar en uso de descanso, entre las cinco categorías.

Lo anterior sugiere que a pesar de que las escuelas cuentan con distintos escenarios, los cortos tiempos de descanso no permiten a los alumnos acudir a sitios distantes que podrían tener mejores cualidades restauradoras. Es preciso mencionar que los espacios al exterior son utilizados por algunos alumnos inscritos en actividades deportivas como futbol o basquetbol. Por ello, se propone que los estudios posteriores que aborden la restauración psicológica de los espacios escolares consideren las diferencias de acceso y tiempo que implican estas actividades deportivas, en las cuales solamente se involucran algunos estudiantes.

Mejía-Castillo y Lagunes-Córdoba (2015) encontraron que los pasillos o corredores afuera del salón son espacios donde una gran parte de los alumnos pasa su tiempo de descanso. En el estudio se encontró que estos escenarios permiten la percepción de extensión, y por lo tanto contri- 
buyen de manera apreciable a la percepción de potencial restaurador total. Los hallazgos aquí presentados confirman que esos espacios son ampliamente utilizados por los estudiantes, ocupando el segundo lugar en uso de espacios de descanso, solo después de los salones de clase. Sin embargo, sus cualidades restauradoras percibidas están por debajo de espacios como las áreas verdes y las explanadas.

En cuanto al análisis por dimensiones específicas de las cualidades restauradoras, la dimensión de compatibilidad no presentó diferencias estadísticamente significativas por el tamaño de la escuela, la extensión de área verde y las variables sociodemográficas. Dado que esta escala se conformó principalmente con reactivos acerca de la posibilidad de relación con los otros en sus lugares de descanso, se sugiere que un espacio físico se vuelve compatible en tanto existan posibilidades de interacción social, sin importar el tipo de escenario de descanso, tamaño, extensión de área verde y sexo de los participantes. En este sentido, se debe tener en cuenta que la adolescencia es una etapa en la que el grupo de pares es muy importante como apoyo social y promotor del bienestar psicológico de las personas (Orcasita y Uribe, 2010). Por ello, los jóvenes necesitan espacios escolares que contribuyan a la interacción con los otros. Los comentarios complementarios al estudio que describen los espacios de descanso aluden continuamente a esta relación: "la cancha es un lugar grande donde convivo con mis amigos y conocidos, tiene bancas donde nos podemos sentar", "[el espacio verde de descanso] es muy bonito ya que hacemos muchos amigos en el lugar, está muy tranquilo y limpio".

Los resultados de diferencias por variables sociodemográficas en las percepciones de cualidades restauradoras muestran que los espacios educativos favorecen la percepción de los estudiantes hombres, quienes tienen puntuaciones más altas que las mujeres en las dimensiones de fascinación, posibilidad de estar alejado y extensión. Algunas investigaciones previas postulan que existen diferencias en el uso del espacio de acuerdo con el sexo, tanto en los escenarios escolares (Ozdemir y Yilmaz, 2008), como en otros espacios públicos (Legendre, 2007). Sin embargo, todavía hace falta mayor investigación sobre las características de las actividades preferidas y uso diferenciado del espacio en adolescentes de escuelas mexicanas. Desde la sociología también se han generado explicaciones acerca del uso inequitativo del espacio entre hombres y mujeres (Cantón, 2007; Shilling, 1991), por lo cual en estudios posteriores sería conveniente involucrar modelos teóricos desde esta perspectiva.

Otra variable sociodemográfica que presenta diferencias significativas es la edad. Los estudiantes más grandes perciben mayores cualidades restauradoras en la escuela.
En este sentido, dado que se recolectaron datos de dos generaciones de ingreso a la escuela, es probable que el tiempo que han pasado los alumnos contribuya a esta percepción positiva; sin embargo, se necesitan estudios específicos para decidir al respecto.

Según la Asociación Mexicana de Agencias de Investigación de Mercados y Opinión Pública ([AMAI], LópezRomo, 2011), el nivel de estudios del jefe de familia es un indicador del nivel socioeconómico. Los resultados aquí presentados muestran que los alumnos con jefas de familia con grados de escolaridad inferior (por lo tanto, de niveles socioeconómicos más bajos) obtienen mayores niveles de percepción de cualidades restauradoras. La Teoría General de la Adaptación (Nelson, 1964) explica que las personas se adecúan a ciertos ambientes cotidianos, lo que podría contribuir a explicar el hecho de que provenir de espacios con carencias y deficiencias físicas contribuiría a una percepción favorable de los centros educativos que forman parte de este estudio.

Con respecto a la preferencia por el lugar de descanso, los resultados de este trabajo confirman que en los lugares favoritos se percibió mayor potencial restaurador que en los no favoritos, tal como lo reportan otros autores y en otros contextos (Korpela, Kytta y Hartig, 2002; MartínezSoto y Montero, 2010; Wilkie y Stavridou, 2013). Es preciso mencionar que varios alumnos de distintas escuelas reportan como espacios favoritos una gran diversidad de lugares (bibliotecas, salas de actos, áreas de lectura, entre otros). Sin embargo, por cuestiones de reglamento, la mayoría de ellos no tiene la posibilidad de pasar su tiempo de descanso ahí.

El tamaño de la escuela es una variable que también se destaca en este estudio. Las escuelas grandes tienen mayores puntuaciones que las pequeñas en fascinación, estar alejado y potencial restaurador total. En cuanto a la fascinación, se infiere que en un espacio amplio puede existir una mayor diversidad de estímulos y situaciones que atraen la atención involuntaria de los alumnos y mayores posibilidades de distanciarse de aquello que demanda atención voluntaria. Con respecto a la posibilidad de estar alejado, las escuelas grandes permitirían que los estudiantes puedan aislarse, durante su tiempo libre, de aquello que les genera tensión.

Finalmente, existe una evidente diferencia en la percepción de cualidades restauradoras entre escuelas con y sin áreas verdes. Sin embargo, al comparar a las escuelas del grupo 3 (50\% a 75\% de extensión de área verde) y 4 (más del 75\% de extensión de área verde), el primer grupo presentó mayor percepción de cualidades restauradoras. Esto no posee una explicación simple y nuestros datos no nos permiten atribuir estas diferencias a una situación concre- 
ta. Es posible que además de la extensión del área verde, el cuidado, el número y tamaño de árboles y arbustos, la presencia de basura, y otras, puedan influir en la percepción de cualidades restauradoras del área verde; estos detalles deberían estudiarse en investigaciones posteriores.

El trabajo aquí presentado nos brinda un panorama acerca de las condiciones en que se encuentran los centros educativos de bachillerato de una ciudad mexicana, y se encontró que las escuelas estudiadas tienen características muy diversas. El análisis con las dimensiones de la Teoría de la Restauración de la Atención permite concluir que los espacios escolares estudiados pueden favorecer la restauración psicológica de los estudiantes, y en general, contribuir al bienestar de estos. Algunos espacios preferidos a los cuales los alumnos no pueden acceder (como bibliotecas, algunas áreas verdes, entre otros) podrían tener este papel de ambientes restauradores y promotores de salud mental. El estudio demuestra que las áreas verdes con mantenimiento y los espacios amplios posibilitan percibir mayor potencial restaurador en las escuelas y, por tanto, nos permite afirmar que estas características pudieran contribuir a la recuperación cognitiva y afectiva de los estudiantes.

Finalmente, los datos obtenidos pueden ser de utilidad para que las instancias correspondientes analicen las condiciones en que se encuentran los centros educativos, y propongan soluciones a las limitaciones espaciales y temporales que pueden afectar la recuperación psicológica de los alumnos.

\section{REFERENCIAS}

Cantón, I. (2007). El espacio educativo y las referencias al género. Revista Interuniversitaria de Formación del Profesorado, 21(2-3), 115-135.

Collado, S. y Corraliza, J. A. (2013). Restauración percibida y pro-ambientalidad infantil. Congreso Internacional de Psicología Ambiental. Barcelona.

Estrada, C. (2007). Efectos psicológicos de la contaminación por ruido en escenarios educativos. Disertación de tesis doctoral. México, D.F.: Universidad Nacional Autónoma de México.

Estrada, C. y Méndez, I. (2010). Impacto del ruido ambiental en estudiantes de educación primaria de la Ciudad de México. Revista Latinoamericana de Medicina Conductual, 1, 57-68.

Han, K. (2009). Influence of Limitedly Visible Leafy Indoor Plants on the Psychology, Behavior, and Health of Students at a Junior High School in Taiwan. Environment and Behavior, 41(5), 567-592.

Hartig, T. y Staats, H. (2003). Guest Editors' Introduction: Restoration environments. Journal of Environmental Psychology, 23(2), 103-107.
Hartig, T., Korpela, K., Evans, G. W. y Garling, T. (1997). A measure of restorative quality of environments. Scandinavian Housing y Planning Research, 14, 175-194.

Hartig, T., Mang, M. y Evans, G. (1991). Restorative Effects of Natural Environment Experiencies. Environment and Behavior, 23(1), 3-26.

Hernández, B. y Hidalgo, M. d. (2005). Effect of urban vegetation on psychological restorativeness. Psychological Reports, 96, 1025-1028.

Instituto Nacional de Estadística y Geografía. (2010). Censo de Población y Vivienda. Recuperado de http://www.censo2010. org.mx/

Kaplan, R. y Kaplan, S. (1989). The experience of nature: a psychological perspective. Nueva York: Cambridge University Press.

Kaplan, S. (1995). The restorative benefits of nature: toward an integrative framework. Journal of Environmental Psychology, $15,169-182$.

Kaplan, S. y Kaplan, R. (2009). Creating a larger role for environmental psychology: The reasonable person model as an intergrative framework. Journal of Environmental Psychology, 29, 329-339.

Kaplan, S., y Talbot, J. (1983). Psychological benefits of a wilderness experience. In I. Altman, y J. F. Wohlwill, Behavior and de natural environment (pp. 163-203). Nueva York: Plenum Press.

Kels, C., Evans, G. y Röderer, K. (2013). The Restorative Effects of Redesigning the Schoolyard: a Multi-Methodological, Quasi-Experimental Study in Rural Austrian Middle Schools. Environment and Behavior, 20(5), 1-21.

Korpela, K., Kytta, M. y Hartig, T. (2002). Restorative experience, self regulation, and children's place preferences. Journal of Environmental Psychology, 22, 287-398.

Kort, Y., Gal, R. y Staats, H. (2004). Restoration in place: the impact of social context and type of setting on restorative effects. (p. 152). Viena, Austria.

Legendre, A. (2007). Gender differences in the development of children's knowledge and use of outdoor public spaces. A case study in a small town of the Paris suburb. Environmental Psychology Conference. Bayreuth.

López-Romo, H. (2011). 21 preguntas frecuentes en relación con el nivel socioeconómico AMAI. Datos diagnósticos y tendencias. La revista de la AMAI, 8-12.

Martínez-Soto, J. y Montero, M. (2010). Percepción de cualidades restauradoras y preferencia ambiental. Revista Mexicana de Psicología, 27(2), 183-190.

Matsuoka, R. (2010). Student Performance and High School Ladscapes: Examining the links. Journal of Ladscape and Urban Planning, 97(4), 273-282.

Mejía-Castillo, A. y Lagunes-Córdoba, R. (2015). Cualidades restauradoras de lugares de descanso en escuelas de Xalapa, México. Congreso de Psicología Ambiental. Granada, España. 
Mejía-Castillo, A. (2010). Estrés ambiental e impacto de los factores estresores en la escuela. Pampedia (7), 3-18.

Nelson, H. (1964). Adaptation-level theory. Nueva York: Harper and Row.

Orcasita, L. y Uribe, A. (2010). La importancia del apoyo social en el bienestar de los adolescentes. Psychologia, Avances de la disciplina, 4(2), 69-82.

Ozdemir, A., y Yilmaz, O. (2008). Assessment of outdoor school environments and physical activity in Ankara's primary schools. Journal of Environmental Psychology, 28, 287-300.

Reyes-Lagunes, I. (1993). Redes semánticas para la construcción de instrumentos. Revista de Psicología Social y Personalidad, 9(1), 81-97.

Scopelliti, M. y Giuliani, M. V. (2004). Choosing restorative environments across the lifespan: A matter of place experience. Journal of Environmental Psychology, 24, 423-437.
Shilling, C. (1991). Social space, gender inequalities and educational differentiation. Brtish Journal of Sociology of Education, 12(1), 23-44.

Urbina, S. (1981). Investigación experimental de algunos factores ambientales de los centros de desarrollo infantil y su influencia e las interacciones sociales y el involucramiento de actividades académicas. Tesis de maestría no publicada. México, D.F.: Universidad Nacional Autónoma de México.

Van den Berg, A., Hartig, A. y Staats, H. (2007). Preference for nature in urbanized societies: stress, restoration and the pursuit of sustainability. Journal of Social Issues, 63(1), 79-96.

Van den Berg, A. y Heijne, M. (2005). Fear versus fascination: Emotional responses to natural threats. Journal of Environmental Psychology, 25(3), 261-272.

Wilkie, S. y Stavridou, A. (2013). Influence of environmental preference and environment type congruence on judgments of restoration potential. Urban Foresty y Urban Greening, 13(2), 163-170. 